\title{
Functional analysis of RNA motifs essential for BC200 RNA-mediated translational regulation
}

\author{
Seonghui Jang ${ }^{1,2}$, Heegwon Shin ${ }^{1} \mathcal{E}$ Younghoon Lee, ${ }^{1, *}$ \\ ${ }^{1}$ Department of Chemistry, KAIST, Daejeon 34141, ${ }^{2}$ Korea Food Research Institute, Wanju 55365, Korea
}

\begin{abstract}
Brain cytoplasmic 200 RNA (BC200 RNA) is proposed to act as a local translational modulator by inhibiting translation after being targeted to neuronal dendrites. However, the mechanism by which BC200 RNA inhibits translation is not fully understood. Although a detailed functional analysis of RNA motifs is essential for understanding the BC200 RNA-mediated translation-inhibition mechanism, there is little relevant research on the subject. Here, we performed a systematic domain-dissection analysis of BC200 RNA to identify functional RNA motifs responsible for its translationalinhibition activity. Various RNA variants were assayed for their ability to inhibit translation of luciferase mRNA in vitro. We found that the 111-200-nucleotide region consisting of part of the Alu domain as well as the A/C-rich domain (consisting of both the A-rich and C-rich domains) is most effective for translation inhibition. Surprisingly, we also found that individual A-rich, A/C-rich, and Alu domains can enhance translation but at different levels for each domain, and that these enhancing effects manifest as cap-dependent translation. [BMB Reports 2020; 53(2): 94-99]
\end{abstract}

\section{INTRODUCTION}

Noncoding RNAs (ncRNAs) are not translated into proteins, but instead participate in regulating various cellular metabolic processes $(1,2)$. ncRNAs exert their diverse functions by forming specific structures capable of serving as signals, guides, scaffolds, or decoys (3-5). Brain cytoplasmic RNAs (BC RNAs) - BC1 RNA in rodents (6) and BC200 RNA in primates $(7,8)$ - act as translational repressors in neurons. In addition to expression in neuronal cells, BC200 RNA is also highly expressed in some cancer cells (9-12). In this context, it has recently been reported that $\mathrm{BC} 200$ RNA can serve as a

*Corresponding author. Tel: +82-42-350-2832; Fax: +82-42-3502810; E-mail: Younghoon.Lee@kaist.ac.kr

https://doi.org/10.5483/BMBRep.2020.53.2.153

Received 4 June 2019, Revised 12 June 2019, Accepted 13 June 2019

Keywords: A/C-rich domain, Alu domain, BC200 RNA, RNA motifs, Translational regulation therapeutic target and diagnostic marker for cancer (10, 13-16).

Although BC1 RNA and BC200 RNA have similar functions and evolutionarily conserved secondary structures, they originate from different ancestors, with $\mathrm{BC} 1$ arising from tRNA $^{\text {Ala }}$ (17) and BC200 RNA arising from SRP (signal recognition particle) RNA (7). Whereas functional motifs of BC1 RNA are relatively well defined, those of BC200 remain poorly understood (18). BC200 RNA consists of three domains: a 5' Alu domain, a central A-rich domain, and a 3' C-rich domain (7). The Alu domain is $89 \%$ homologous to the left monomer of the Alu-J repetitive element present in the human genome (7). The downstream promoter elements (A-box and B-box) for RNA polymerase, which are important for binding of the heterodimeric protein SRP9/14 $(7,19)$, lie in the Alu domain (11). BC200 RNA has been reported to directly interact with SRP9/14 in vitro and in vivo (19), but the role of the BC200 ribonucleoprotein (RNP) of BC200 RNA and SRP9/14 in translation inhibition has not been fully elucidated. The A-rich domain of BC200 RNA is responsible for interacting with poly $\mathrm{A}$ binding protein (PABP) and the eukaryotic translation initiation factor elF4A in vitro as well as in vivo $(20,21)$. The interaction of PABP with BC200 RNA has been proposed to result in BC200 RNA-mediated translation inhibition by interfering with the translation-activating function of PAPB $(21,22)$. Binding of BC200 RNA to elF4A inhibits the helicase activity of elF4A, thereby inhibiting translation initiation $(20,23)$. Hence, the A-rich domain has a major role in the translation-inhibition activity of BC200 RNA. The C-rich domain provides a binding site for elF4B, and the BC 200 RNA-elF4B interaction prevents the binding of elF4B to $18 \mathrm{~S}$ rRNA (23). Phosphorylation of elF4B also affects the binding affinity of BC200 RNA for elF4B (24). Therefore, the A-rich and $\mathrm{C}$-rich domains appear to be indispensable for translation inhibition by BC200 RNA. However, the role of the Alu domain in BC200 RNA-mediated translation inhibition remains unclear. Moreover, a detailed dissection of BC200 RNA structure and the respective effects of domains is still lacking.

In this study, we produced a variety of systematically generated BC200 RNA variants and examined their respective functions in translational regulation. We found that the A/C-rich domain (consisting of both the A-rich and C-rich 
domains) and part of the Alu domain are required for effective translation inhibition. We also found that A-rich, A/C-rich, and Alu domains enhance translation in a cap-dependent manner.

\section{RESULTS}

\section{Dissecting RNA motifs}

We generated 3'-serially deleted BC200 RNAs and analyzed their effects on translation in in vitro translation assays using a coupled transcription/translation reticulocyte system (Fig. 1). Deletion to position 162, generating BC200(1-161) lacking the C-rich domain, caused only a modest decrease in BC200 RNA-mediated translation inhibition. Further deletion to position 122, generating BC200(1-121) lacking both the A-rich domain and C-rich domain, led to a complete loss of BC200 RNA translation-inhibition ability, suggesting that the A-rich domain is most effective in BC200 RNA-mediated translation inhibition, whereas the C-rich domain plays a minor role. We also performed a $5^{\prime}$-deletion analysis (Fig. 2A and B). Deletion of the 5'-most 24 nucleotides, yielding BC200(25-200), slightly weakened the translation-inhibition activity of BC200 RNA. Deletion to position 80, generating BC200(81-200), had little significant effect on translation inhibition, but additional deletion to position 121, generating BC200(122-200), caused a

\section{A}

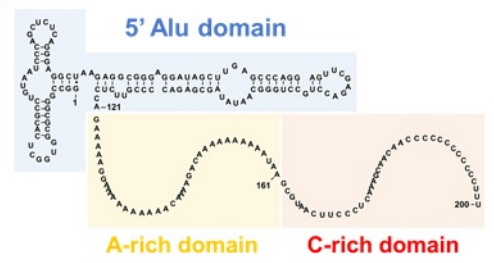

B

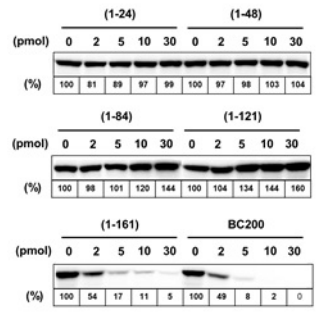

C

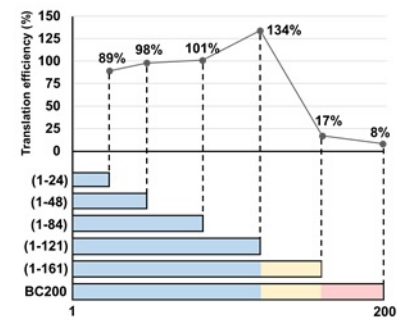

Fig. 1. Effects of $3^{\prime}$-deletion variants of $B C 200$ RNA on in vitro translation. (A) Schematic diagram of BC200 RNA structure. (B) In vitro translation of luciferase mRNA in the presence of increasing amounts of $3^{\prime}$ deletion variants of BC200 RNA was analyzed in a coupled in vitro transcription/translation system and the levels of the expressed luciferase protein were analyzed on an SDS-PAGE gel. The ratio $(\%)$ of protein levels in the presence versus absence of RNA are presented for relative translational efficiencies. (C) Ratios of protein levels in the presence versus absence of BC200 RNA are plotted against deleted regions. severe effect, suggesting that the sequence between positions 81 and 121, corresponding to resides within the Alu domain, is essential for effective BC200 RNA-mediated translation inhibition. A more detailed analysis of this region showed that the sequence between positions 111 and 121 contains a sequence that is crucial for translation inhibition (Fig. 2C and D). Further deletion of sequences between position 122 and 161 comprising the A-rich domain caused a loss of BC200 RNA translation-inhibition ability. The 5'-deletion analysis suggests that the $3^{\prime}$-most 11 nucleotides of the Alu domain and the entire A-rich domain play important roles in BC200 RNA-mediated translation inhibition, and that the C-rich domain and the $5^{\prime}$-most 24 nucleotides of the Alu domain play a minor role.

Next, we generated and analyzed the following domain variants of BC200 RNA: BC200(122-161), comprising the A-rich domain; $\mathrm{BC} 200 \Delta(122-161)$, lacking the A-rich domain; BC200(1-121), comprising the Alu domain; and BC200(122-200), comprising both $\mathrm{A}$ - and $\mathrm{C}$-rich domains (A/C-rich domain) (Fig. 3). Translation inhibition increased with increasing amounts of the A-rich domain, but the efficacy of the resulting inhibition was less than that of the A/C-rich domain and much less than that of full-length BC200 RNA. It thus seems likely that the A-rich domain requires other RNA motifs, such as the C-rich domain, as well as the $3^{\prime}$-most 11 nucleotides and $5^{\prime}$-most 24 nucleotides of the Alu domain as mentioned above, for
A

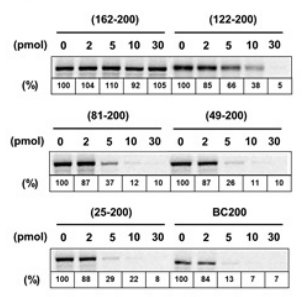

C

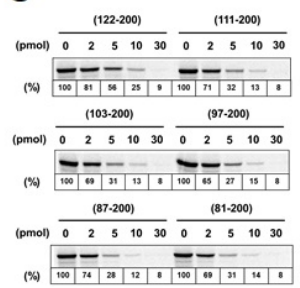

B

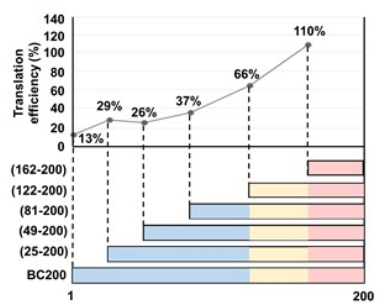

D

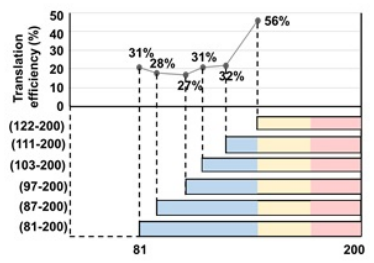

Fig. 2. Effects of $5^{\prime}$-deletion variants of $B C 200$ RNA on in vitro translation. (A, C) In vitro translation of luciferase mRNA in the presence of increasing amounts of $5^{\prime}$-deletion variants of BC200 RNA was analyzed in a coupled in vitro transcription/translation system as in Fig. 1. (B, D) Ratios of protein levels in the presence versus absence of 0.5 pmol of BC200 RNA variants are plotted against deleted regions. 
A

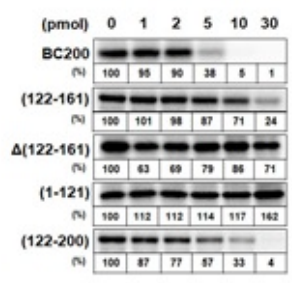

D

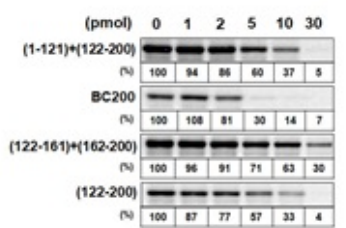

B

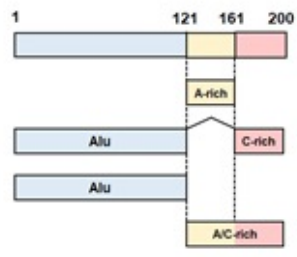

E

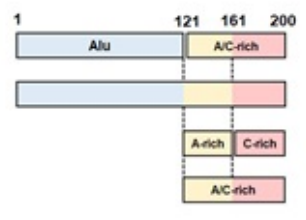

C

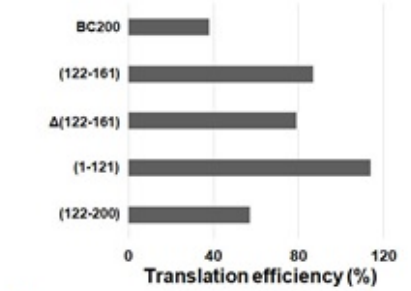

$\mathbf{F}$

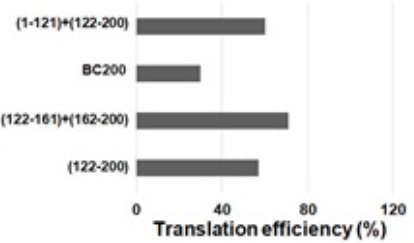

Fig. 3. Effects of domain variants of BC200 RNA on in vitro translation. (A, D) In vitro translation of luciferase mRNA in the presence of increasing amounts of domain variants of $\mathrm{BC} 200$ RNA was analyzed in a coupled in vitro transcription/translation system. Cases where mixtures of two RNA variants were used are denoted by insertion of " + " between each variant. (B, E) Schematic diagram of domain variants of BC200 RNA. (C, F) Ratios of protein levels in the presence versus absence of $0.5 \mathrm{pmol}$ of $\mathrm{BC} 200$ RNA variants are presented as translation efficiency (\%). effective translation inhibition. We next tested whether a mixture of the Alu domain and A/C-rich domain had the same effect as full-length BC200 RNA, but found that this mixture only showed an inhibitory effect comparable to that of the A/C-rich domain (Fig. 3A-C). Again, the inhibitory effect of a mixture of the A-rich domain and C-rich domain on translation was less than that of the A/C-rich domain (Fig. 3D-F). These results suggest that Alu, A-rich, and C-rich domains cooperatively participate in inhibiting translation only when linked in a single molecule.

Interestingly, we also found that BC200(1-84) and BC200(1-121) slightly enhanced translation at a low concentration (5 pmol), and produced more profound enhancing effects at higher concentrations (Fig. 1B and C). Translation enhancement by the Alu domain was previously shown (25); here, we more sharply defined the region responsible for the Alu domainmediated translation-activation as the sequence between positions 1 and 84 .

\section{Effects of BC200 RNA variants on cap/poly(A)-dependent translation}

Because we used a coupled transcription/translation system for in vitro translation assays, it is possible that the effects of BC200 RNA variants on translation described above were limited to unmodified luciferase reporter mRNAs. To test this, we performed in vitro translation assays in a standard reticulocyte lysate system using in vitro-transcribed mRNAs without modification or bearing a $5^{\prime}$ m7G-cap and/or a $3^{\prime}$ poly(A) tail (Fig. 4). We found that the effects of full-length BC200 RNA, BC200(111-200), and BC200(162-200), i.e. the C-rich domain, were not significantly affected by the mRNA-modification state: full-length BC200 RNA and
A

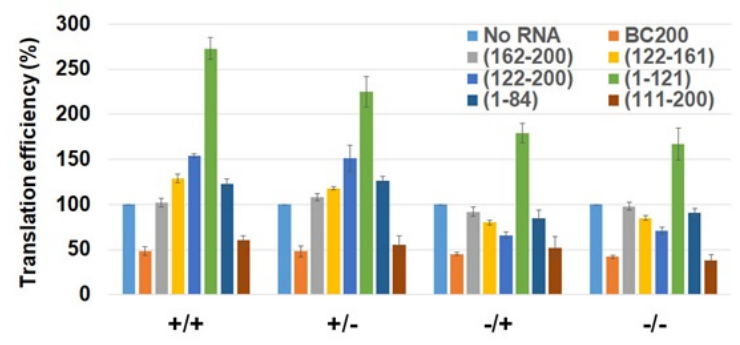

B

\begin{tabular}{|c|c|c|c|c|c|}
\hline & $+/+$ & $+/-$ & $-/+$ & $-/-$ & Description \\
\hline BC200 & $\downarrow$ & $\downarrow$ & $\downarrow$ & $\downarrow$ & Full length \\
\hline$(\mathbf{1 6 2 - 2 0 0 )}$ & - & - & - & - & C-rich \\
\hline$(122-161)$ & $\uparrow$ & $\uparrow$ & $\downarrow$ & $\downarrow$ & A-rich \\
\hline$(122-200)$ & $\uparrow$ & $\uparrow$ & $\downarrow$ & $\downarrow$ & A/C-rich \\
\hline$(1-121)$ & $\uparrow$ & $\uparrow$ & $\uparrow$ & $\uparrow$ & Alu \\
\hline$(1-84)$ & $\uparrow$ & $\uparrow$ & - & - & Part of Alu \\
\hline$(111-200)$ & $\downarrow$ & $\downarrow$ & $\downarrow$ & $\downarrow$ & $\begin{array}{c}\text { Part of Alu }+ \\
\text { A/C-rich }\end{array}$ \\
\hline
\end{tabular}

Fig. 4. Different effects of $B C 200$ RNA variants on in vitro translation according to mRNA modification state. (A) In vitro translation of luciferase mRNA was analyzed in a standard reticulocyte lysate system containing 0.5 pmol of BC200 RNA variants. Modification states of luciferase mRNAs used are as follows: $+/+$, $\operatorname{cap}+/ \operatorname{poly}(\mathrm{A})+$; $+/-$, $\operatorname{cap}+/ \operatorname{poly}(\mathrm{A})-$; $-/+$, cap $-/ \operatorname{poly}(\mathrm{A})+;-/-$, cap $-/ \operatorname{poly}(\mathrm{A})-$. '(B) Ratios of protein levels in the presence versus absence of BC200 RNA variants were evaluated and defined as increased $(\uparrow)$, decreased $(\downarrow)$ or similar levels $(-)$ according to the criterion of $>1.2$-fold change. 
BC200(111-200) led to translation inhibition, whereas the $\mathrm{C}$-rich domain had little effect on translation. The presence or absence of poly $(\mathrm{A})$ tails made little difference in the effects of BC200 RNA variants on translation. However, translation was enhanced by the Alu domain in the presence of a $5^{\prime}$ cap. Furthermore, translational activation by BC200(1-84), which required a high concentration in the absence of the $5^{\prime}$ cap, was observed at a concentration of 5 pmol with $5^{\prime}$-capped mRNA. Interestingly, the A-rich domain and A/C-rich domain activated translation when a 5'-capped mRNA was used. This contrasts with the translation-inhibitory effects of these domains in the absence of the $5^{\prime}$ cap. Taken together, these results suggest that translation activation by RNA motifs of BC200 RNA is greatest with mRNAs bearing a 5' cap.

\section{DISCUSSION}

In the current study, we showed that the most effective region of BC200 RNA for translation inhibition corresponds to the sequence from position 111 to 200 . This region contains the 3'-most 11 nucleotides of the Alu-domain as well as A-rich and C-rich domains. The Alu, A-rich, and C-rich domains cooperatively contribute to BC200 RNA-mediated translation inhibition only when they are arranged in cis in a single molecule.

We also found that the individual A-rich, A/C-rich, and Alu domains can actually enhance translation, and that these enhancing effects are greatest with mRNAs bearing a $5^{\prime}$ cap. It was previously reported that free poly $(\mathrm{A})$ stimulates translation with 5'-capped mRNAs, but inhibits translation with uncapped mRNA (26). The stimulatory effect reflects elF4G-PABP interactions, which increase the affinity of elF4E for the $5^{\prime}$ cap. Therefore, it is likely that the individual A-rich domain of BC200 RNA affects translation through a similar mechanism. Alu RNA and 7SL RNA were reported to stimulate translation, although the specific regions responsible for the stimulatory effects were not defined $(27,28)$. The Alu domain of BC200 RNA may have a function similar to that of Alu RNA and 7SL RNA in translational regulation. However, RNPs of 7SL RNA and Alu RNA containing SRP 9/14 inhibit translation $(27,28)$. BC200 RNA also interacts with SRP9/14 in vitro and in vivo $(19,29)$, although the role of BC200 RNPs containing SRP9/14 remains unclear. Future studies should determine how BC200 RNPs, such as BC200 RNA-SRP9/14, are involved in translational regulation.

BC200 RNA may differentially regulate translation by distinguishing among mRNAs base on their $5^{\prime}$ capping state. Accordingly, BC200 RNA could alter gene expression patterns in very specific ways. A recent ribosome profiling analysis showed that, of 29 genes whose ribosomal occupation was altered more than 2-fold by BC200 knockdown, 16 were upregulated and 13 were downregulated (30). The fact that BC200 RNA alters only a limited number of genes in vivo suggests the existence of an additional mechanism that confers such specificity in vivo. Protein binding to BC200 RNA may contribute to modulation of the translation-regulating activity of BC200 RNA. Because BC200 RNA occupies very specific subcellular sites in neuronal or cancer cells $(7,31)$, distinct localization of BC200 RNA may also play an important role in BC200 RNA-mediated translational regulation.

The observation that the BC200 RNA molecule contains RNA motifs that provide negative and positive effects on translation is intriguing. Because full-length BC200 RNA has translation-repressing activity, the translation-activating activity of individual RNA motifs may not occur in cells. However, if translation-repressing RNA motifs, such as the A-rich domain, are veiled by cellular factors, such as proteins or other RNAs, it is possible that displacing these factors could allow the translation-activating RNA motifs to exert their activity. In this latter context, it has been suggested that other BC200 RNA-binding proteins can hinder protein binding to a specific BC200 RNA motif (12). Notably, it has also been reported that hnRNP E1 and E2 restore BC200 RNA-inhibited translation by binding the C-rich domain (32). Therefore, it is possible that individual RNA motifs may be differently involved in BC 200 RNA-mediated translational regulation in a cell-type-specific and spatially distinct manner.

\section{MATERIALS AND METHODS}

\section{In vitro transcription}

The DNA templates for in vitro transcription were constructed by polymerase chain reaction (PCR) using the primer pairs shown in Table S1. In vitro transcripts were synthesized using RiboMAX Large Scale RNA Production Systems (Promega) and gel-purified. Poly $(A)+m R N A$ and poly $(A)-m R N A$ were prepared as previously described (25). The 5'-capped mRNAs were synthesized by adding Ribo $\mathrm{m}^{7} \mathrm{G}$ Cap Analog (Promega) to in vitro transcripts, according to the manufacturer's protocol.

\section{In vitro translation}

For in vitro translation, a TnT (transcription/translation)-coupled rabbit reticulocyte lysate system (Promega, L4610) or a standard rabbit reticulocyte lysate system (Promega, L4960) was used. Reaction mixtures (total volume, $10 \mu \mathrm{l}$ ) containing $\left({ }^{35} \mathrm{~S}\right)$ methionine, buffer, amino acids mixture (minus methionine), increasing amounts of BC200 RNA or its variants, and $0.2 \mu \mathrm{g}$ luciferase expression plasmid DNA (TnT-coupled rabbit reticulocyte lysate system) or $0.5 \mu \mathrm{g}$ luciferase mRNA (standard rabbit reticulocyte lysate system) were incubated for $90 \mathrm{~min}$ at $30^{\circ} \mathrm{C}$ as recommended by the manufacturer. Reaction mixtures were electrophoresed on sodium dodecyl sulfate-polyacrylamide gels. Gels were analyzed using a phospho-image analyzer (FLA-7000; Fuji), and protein bands were quantified using Image J software (NIH). 


\section{ACKNOWLEDGEMENTS}

This study was funded by National Research Foundation of Korea (NRF) Grants from the Korean government (MSIT) (2017R1A6A3A11031308; 2019R1H1A2039730) and by KAIST.

\section{CONFLICTS OF INTEREST}

The authors have no conflicting interests.

\section{REFERENCES}

1. Strobel EJ, Watters KE, Loughrey D and Lucks JB (2016) RNA systems biology: uniting functional discoveries and structural tools to understand global roles of RNAs. Curr Opin Biotechnol 39, 182-191

2. Cech TR and Steitz JA (2014) The noncoding RNA revolution-trashing old rules to forge new ones. Cell 157, 77-94

3. Wan $Y$, Kertesz $M$, Spitale RC, Segal E and Chang HY (2011) Understanding the transcriptome through RNA structure. Nat Rev Genet 12, 641-655

4. Washietl S (2010) Sequence and structure analysis of noncoding RNAs. Methods Mol Biol 609, 285-306

5. Johnsson P, Lipovich L, Grandér D and Morris KV (2014) Evolutionary conservation of long non-coding RNAs; sequence, structure, function. Biochim Biophys Acta 1840, 1063-1071

6. DeChiara TM and Brosius J (1987) Neural BC1 RNA: cDNA clones reveal nonrepetitive sequence content. Proc Nat Acad Sci U S A 84, 2624-2628

7. Tiedge H, Chen W and Brosius J (1993) Primary structure, neural-specific expression, and dendritic location of human BC200 RNA. J Neurosci 13, 2382-2390

8. Skryabin BV, Kremerskothen J, Vassilacopoulou D et al (1998) The BC200 RNA gene and its neural expression are conserved in Anthropoidea (Primates). J Mol Evol 47, 677-685

9. Vikram R, Ramachandran R and Abdul K (2014) Functional significance of long non-coding RNAs in breast cancer. Breast Cancer 21, 515-521

10. Hu T and Lu YR (2015) BCYRN1, a c-MYC-activated long non-coding RNA, regulates cell metastasis of non-small-cell lung cancer. Cancer Cell Int 15, 36

11. Kim Y, Lee J, Shin H, Jang S, Kim SC and Lee Y (2017) Biosynthesis of brain cytoplasmic 200 RNA. Sci Rep 7, 6884

12. Jung E, Lee J, Hong HJ, Park I and Lee Y (2014) RNA recognition by a human antibody against brain cytoplasmic 200 RNA. RNA 20, 805-814

13. Zhao RH, Zhu CH, Li XK et al (2016) BC200 LncRNA a potential predictive marker of poor prognosis in esophageal squamous cell carcinoma patients. Onco Targets Ther 9, 2221-2226

14. Wu DI, Wang T, Ren C et al (2016) Downregulation of BC200 in ovarian cancer contributes to cancer cell proliferation and chemoresistance to carboplatin. Oncol Lett 11, 1189-1194
15. Zhang XY, Zhang LX, Tian CJ et al (2016) LncRNAs BCYRN1 promoted the proliferation and migration of rat airway smooth muscle cells in asthma via upregulating the expression of transient receptor potential 1. Am J Transl Res 8, 3409-3418

16. Shin H, Kim Y, Kim M and Lee $Y$ (2018) BC200 RNA: An emerging therapeutic target and diagnostic marker for human cancer. Mol Cells 41, 993-999

17. Rozhdestvensky TS, Kopylov AM, Brosius J and Huttenhofer A (2001) Neuronal BC1 RNA structure: evolutionary conversion of a $\mathrm{RNA}(\mathrm{Ala})$ domain into an extended stem-loop structure. RNA 7, 722-730

18. Sosinska P, Mikula-Pietrasik J and Ksiazek K (2015) The double-edged sword of long non-coding RNA: The role of human brain-specific BC200 RNA in translational control, neurodegenerative diseases, and cancer. Mutat Res Rev Mutat Res 766, 58-67

19. Kremerskothen J, Zopf D, Walter $\mathrm{P}$ et al (1998) Heterodimer SRP9/14 is an integral part of the neural BC200 RNP in primate brain. Neurosci Lett 245, 123-126

20. Lin D, Pestova TV, Hellen CU and Tiedge H (2008) Translational control by a small RNA: dendritic BC1 RNA targets the eukaryotic initiation factor $4 \mathrm{~A}$ helicase mechanism. Mol Cell Biol 28, 3008-3019

21. Muddashetty R, Khanam T, Kondrashov A et al (2002) Poly(A)-binding protein is associated with neuronal BC1 and BC200 ribonucleoprotein particles. J Mol Biol 321, 433-445

22. Kahvejian A, Roy G and Sonenberg N (2001) The mRNA closed-loop model: the function of PABP and PABPinteracting proteins in mRNA translation. CSH Symp Quant Biol 66, 293-300

23. Eom T, Berardi V, Zhong J, Risuleo G and Tiedge $\mathrm{H}$ (2011) Dual nature of translational control by regulatory BC RNAs. Mol Cell Biol 31, 4538-4549

24. Eom T, Muslimov IA, Tsokas P et al (2014) Neuronal BC RNAs cooperate with elF4B to mediate activity-dependent translational control. J Cell Biol 207, 237-252

25. Kondrashov AV, Kiefmann M, Ebnet K, Khanam T, Muddashetty RS and Brosius J (2005) Inhibitory effect of naked neural BC1 RNA or BC200 RNA on eukaryotic in vitro translation systems is reversed by poly(A)-binding protein (PABP). J Mol Biol 353, 88-103

26. Borman AM, Michel YM, Malnou CE and Kean KM (2002) Free poly(A) stimulates capped mRNA translation in vitro through the elF4G-poly(A)-binding protein interaction. J Biol Chem 277, 36818-36824

27. Hasler J and Strub K (2006) Alu RNP and Alu RNA regulate translation initiation in vitro. Nucleic Acids Res 34, 2374-2385

28. Rubin CM, Kimura RH and Schmid CW (2002) Selective stimulation of translational expression by Alu RNA. Nucleic Acids Res 30, 3253-3261

29. Bovia F, Wolff N, Ryser S and Strub K (1997) The SRP9/14 subunit of the human signal recognition particle binds to a variety of Alu-like RNAs and with higher affinity than its mouse homolog. Nucleic Acids Res 25, 318-326

30. Shin H, Lee J, Kim Y et al (2017) Knockdown of BC200 RNA expression reduces cell migration and invasion by destabilizng mRNA for calcium-binding protein S100A11. 
BC200 RNA motifs for translational regulation Seonghui Jang, et al.

RNA Biol 14, 1418-1430

31. Shin $H$, Lee J, Kim $Y$, Jang $S$, Ohn $T$, and Lee $Y$ (2017) Identifying the cellular location of brain cytoplasmic 200 RNA using an RNA-recognizing antibody. BMB Rep 50,
318-332

32. Jang S, Shin H, Lee J, Kim Y, Bak G and Lee Y (2017) Regulation of BC200 RNA-mediated translation inhibition by hnRNP E1 and E2. FEBS Lett 591, 393-405 